\title{
Genetic Diversity of Maize (Zea mays L.) Inbred Lines Revealed by Simple Sequence Repeat Markers
}

\author{
Savita Sharma $^{1 *}$, D.P. Mishra1, Bhupender Kumar ${ }^{2}$, Harpreet Kaur ${ }^{2}$ and H.R. Jewlia ${ }^{2}$ \\ ${ }^{1}$ Shri Venkateshwara University, UP, India \\ ${ }^{2}$ ICAR-Indian Institute of Maize Research, New Delhi-110012, India \\ *Corresponding author
}

\section{A B S T R A C T}

\begin{tabular}{|l|}
\hline Ke y w or d s \\
Zea mays, Simple \\
sequence repeat, \\
Genetic diversity, \\
Maize clusters. \\
\hline Article Info \\
\hline Accepted: \\
07 October 2017 \\
Available Online: \\
10 December 2017 \\
\hline
\end{tabular}

\begin{abstract}
A set of 33 maize inbred lines were analyzed using 40 Simple Sequence Repeat (SSR) markers. Out of 40 SSR markers, 25 markers were found reproducible in which 18 were found polymorphic and 7 monomorphic. Total 40 alleles ranges from 2 to 3 with mean of 2.22 alleles per loucs were identified among 33 lines using 18 polymorphic SSR markers. The average polymorphism Information Content (PIC) was 0.36 with range of 0.1 to 0.56 . Unweighted Paired Group Method using Arithmetic Averages (UPGMA) cluster analysis grouped the genotypes into two major clusters. Cluster I was the biggest one with 25 genotypes which was further sub-divided into two sub-clusters with 21 and 4 genotypes, respectively. The major cluster II contained 9 genotypes in two sub clusters each with 7 and 2 genotypes. The identified clusters may find useful to attempt crossing programme in maize breeding programme. In this study, we found SSR as a good complementing tool along with morphological markers for maize genetic characterization.
\end{abstract}

\section{Introduction}

Maize (Zea mays L.), popularly known as the queen of cereals, considered as third most important cereal crop after wheat and rice in the world. India ranks fourth in terms of the maize growing country in the world with 9.4 million hectare area, 24.26 million tonnes of production and 2.57 t/ha average productivity (G.O.I. 2014). Hence, it clearly implies that maize has a unique place in Indian economy. There is wide spread interest towards characterization of genetic diversity among genotypes in various crops species. Maize (Zea mays $\mathrm{L}, 2 \mathrm{n}=20$ ) is one of the important food grain crops, in which heterosis has been widely exploited. Knowledge of the genetic diversity among commercially important maize inbred lines helps hybrid maize breeding programme by planned utilization of promising source germplasm (Pushpavalli et al., 2001 and Stich et al., 2005). It is well established that morphological characterization alone does not reliably depict the genetic relationships among the genotypes due to environmental interactions, largely unknown genetic control of these traits and inadequate sampling of the genome (Reif et al., 2005). Biochemical markers such as isozymes, and chromatographic data of zein, have been extensively used to examine the genetic diversity in commercial maize genotypes (Bar-Hen et al., 1995). However, limited number of available marker loci and 
low level of polymorphism are some of the major limitations of the biochemical markers (Xu et al., 2004).

Lopes et al., (2015) investigated the genetic diversity of twenty-two sweet corn cultivars viz., seventeen open-pollinated varieties (OPV) and five hybrids $(\mathrm{H})$ by applying 30 polymorphic simple sequence repeat markers. They detected 86 alleles with polymorphism information content ranged from 0.19 (umc2319) to 0.71 (umc2205). Average of 2.67 alleles per locus determined in the study emphasizes broadening of sweet corn germplasm.

Ko et al., (2016) investigated the genetic diversity and genetic relationships of 87 super sweet corn inbred lines using SSR and SSAP markers and analyze their population structure. In their study, SSR markers showed higher average gene diversity and Shannon's information index than SSAP markers. Considering the importance of genetic diversity study in maize and utility of SSRs markers as a good tool for this, the present study has been designed with the aim to identify the diverse groups in available materials which will accelerate the hybrids breeding programme.

\section{Materials and Methods}

\section{Plant material}

A set of 33 maize inbred lines (Table 1) with different genetic background was selected from the lines available at ICAR-Indian Institute of Maize Research Pusa Campus, New Delhi.

\section{DNA isolation and quantification}

DNA was extracted from pooled sample of leaves from 15 seedlings following the method (Saghai-Maroof et al., 1984) with minor modifications. One gram fresh leaf sample was used for DNA isolation using a cetyltrimetyl ammonium Bromide (CTAB) extraction method (Stacey and Issac et al., 1994).The samples were grind in autoclaved mortor and pestle to a fine powder with liquid nitrogen. The fine powder was transferred to label $5 \mathrm{ml}$ centrifuge (Micro tube) tube containing $1 \mathrm{ml} \mathrm{CTAB}$ extraction buffer (lysis buffer). Sample tubes (microtubes) were placed in water bath at $65^{\circ} \mathrm{C}$ for $1 \mathrm{hr}$ and gently mixed after every 10-15 minutes. The tubes were removed from water bath and contents were allowed to cool at room temperature. Equal volume of chloroform: isoamylalcohol (24: 1) was added and mixed thoroughly. Upper layer of the centrifuged sample were collected in fresh tubes and 0.6 volume of chilled isopropanol was added and incubated at $-20^{\circ} \mathrm{C}$ for 1 hour to allow precipitation. Samples were centifuged at $10000 \mathrm{rpm}$ for 10 minutes. Supernatant was discarded and pellet was washed twice with $500 \mu \mathrm{l}$ of $70 \%$ ethanol.

Finally pellet was kept at room temperature for $37^{\circ} \mathrm{C}$. After that dried pellet was dissolved in $1 \mathrm{ml} \mathrm{TE}(100 \mathrm{Mm})$ and stored in $4^{0} \mathrm{C}$ for further use. Agarose gel (1\%) electrophoresis was utilized to estimate quantity and quality of DNA. DNA samples for loading were prepared by taking $2 \mu \mathrm{l}$ quantity of DNA. Good quality, uncut lambda DNA of known quality 50ng, 100ng, 200ng, 400ng lambda DNA having concentration of pure DNA as $50 \mathrm{ng} / \mu \mathrm{l}$, was also prepared. The electrophoresis DNA samples were visualized using a UV trans-illuminator gel documentation system.

\section{Primers and PCR amplification}

A set of 40 SSR markers, distributed uniformly across 10 chromosomes were used in the study (Table 1). Out of 40 SSR marker only 25 markers were amplified and found 
reproducible. The PCR amplification cycle consisted of initial denaturation at $94^{\circ} \mathrm{C}$ for 10 min followed by 35 cycles of denaturation at $94^{\circ} \mathrm{C}$ for $1 \mathrm{~min}$, primer annealing at $55^{\circ} \mathrm{C}$ for $30 \mathrm{sec}$ and primer extension at $72^{\circ} \mathrm{C}$ for 1 min. The final extension step was performed at $72^{\circ} \mathrm{C}$ for $1 \mathrm{~min}$. PCR was carried out in a volume of $20 \mu \mathrm{l}$, containing $50 \mathrm{ng}$ of template DNA, $1.5 \mu 1$ of each forward and reverse primers, $0.5 \mathrm{U}$ Taq Polymerase, $0.4 \mathrm{~mm}$ $\mathrm{MgCl}_{2}$. The amplified products were resolved on $3 \%$ agarose gel with 50bp ladder.

\section{SSR data analysis and clustering}

Scoring of the SSR alleles was performed manually in terms of position of bands. Summary for each marker was estimated. The diversity parameters like PIC value and major allele frequency was also estimated.

The SSR data set was subjected estimation of allele frequency which was used to calculate the pairwise distances between individuals. Grouping of genotypes was done using GGT 2.0 software (Berloo, 2008) to produce an agglomerative hierarchical classification by employing Unweighted Paired Group Method using Arithmetic Averages (UPGMA).

The polymorphism information content (PIC) was determined as described by Senior and Henn (1993), which is equal to $1-\Sigma \mathrm{Pij}^{2}$, where $\mathrm{Pij}$ is the frequency of jth allele at ith locus summed across all alleles in the locus. Alleles with frequency of less than 0.20 were considered as rare alleles and such allele representing a particular genotype was considered as unique allele for that genotype.

Jaccard's coefficient (J) (Jaccard et al., 1908) was used to calculate the genetic similarities among pair wise comparison of genotypes based on SSR data, as follows: $\mathrm{J}=\mathrm{N} 11 /$ $(\mathrm{N} 11+\mathrm{N} 10+\mathrm{N} 01)$ where $\mathrm{N} 11$ is the number of bands present in both genotypes; N10 is the number of bands present in one genotype (lane) and N01 the number of bands present in the other genotype. Genetic distances between the genotypes were calculated using 1 - coefficient of genetic similarity.

\section{Results and Discussion}

Total 40 alleles among the 33 lines were identified by 18 polymorphic SSR markers which range from 2 to 3 with an average value of 2.22 (Table 2). Four loci (bnlg108, bnlg1523, phi112 and bnlg128) gave 3 alleles and 14 loci (phi056, bnlg2086, phi038, phi96100, bnlg1940, nc133, umc2104, umc1908, phi085, umc1378, umc1889, umc1161, umc1061 and umc1249) provide 2 alleles. The size of allele ranges from 50400bp (Fig. 1 and 2; Table 2). The polymorphism information content (PIC) ranges from 0.1 to 0.56 with an average of 0.36 (Table 2). Only three markers viz. umc1249, umc1061, and umc1908 provide the highly polymorphic values $(0.56,0.5$ and $0.5)$.

These values are equal to or more than 0.5 , indicating their potential utility to detect differences among the inbred lines. However, three markers viz. provide/give very low PIC value of less than 0.2 and they may not be useful in discriminating inbreds. Those primers revealing more number of monomorphic bands were showing less PIC value and vice versa for primers, which were showing more unique and null alleles.

The resulting dendrogram indicated that all the genotypes could be differentiated and clustered into two major clusters (Fig. 3). Cluster I was the biggest one with 25 genotypes which was further divided into two sub-clusters with 21 and 4 genotypes, respectively. The cluster II contained 9 genotypes in two sub clusters with 7 and 2 genotypes, respectively. 
Table.1 List of Genotypes used for genetic diversity study using simple sequence repeat markers

\begin{tabular}{lll}
\hline S.NO. & Genotype & Source Germplasm \\
\hline 1 & DML-127 & P4855 \\
2 & DML-19 & EC44612 \\
3 & DML-242 & P5133 \\
4 & DML-310 & Seed Tech 2324 \\
5 & DQL-1005 & HQPM1 \\
6 & HK1-42050 & NA \\
7 & UMI-1200 & NA \\
8 & DQL-630-(ORANGE)3-6 & NA \\
9 & DQL-574-2 & NA \\
10 & DQL-593-3 & NA \\
11 & DQL-633-1-1 & NA \\
12 & CM-125 & P45 \\
13 & CML-114 & G25Q \\
14 & CML-172 & P30 \\
15 & CML-220 & P500 \\
16 & CML-312 & P590 \\
17 & CML-334 & P36 \\
18 & CML-40BBB & NPH-28 \\
19 & CML-451XE62 & P43 \\
20 & CML494 & EV7992 \\
21 & CML-207 & EV7992 \\
22 & CML-208BBB & G26Q \\
23 & CML-163 & NA \\
24 & LM-16 & DML177 X BML6 (17) \\
25 & IML13-17 & DML177 X BML6 (22) \\
26 & IML13-22 & DML177 X BML6 (84) \\
27 & IML-13-84 & CML269 X HKI4C4B (7) \\
28 & IML-15-7 & CML269 X HKI4C4B (60) \\
29 & IML-15-60 & CML269 X HKI4C4B (288) \\
30 & IML-15-288 & CML269 X HKI4C4B \\
31 & IML-15-299 & P72clXbrasil1177XESM13 (210) \\
32 & IML-16-210 & \\
33 & IML-16-279 & P72 \\
\hline
\end{tabular}


Table.2 Details of SSR markers used for study of genetic diversity in maize inbred lines

\begin{tabular}{|c|c|c|c|c|c|c|c|}
\hline S. No. & Marker & Forward & Reverse & No. Alleles & An.Tm & PIC & Bin \\
\hline 1 & phi056 & ACTTGCTTGCCTGCCGTTAC & CGCACACCACTTCCCAGAA & 2 & 55 & 0.37 & 1.00 \\
\hline 2 & bnlg 2086 & CGGAACCTGCTGCAGTTAAT & GAGATGCAGGAATGGGAAAA & 2 & 55 & 0.26 & 1.04 \\
\hline 3 & phi038 & TCAGACTCCGCCCAGCAATCA CTG & AGCCTAGTGCTTATCTTGAAGGCTT & 2 & 55 & 0.17 & 1.08 \\
\hline 4 & phi96100 & AGGAGGACCCCAACTCCTG & TTGCACGAGCCATCGTAT & 2 & 55 & 0.49 & 2.01 \\
\hline 5 & bnlg108 & GCAC TCACGCGCACAGGTCA & CGCCTGCCAAGGTACATCAC & 3 & 55 & 0.35 & 2.04 \\
\hline 6 & bnlg1940 & CCTTTTGTTTCAGGCCGTTA & CAGCAGCCTGATGATGAACA & 2 & 55 & 0.42 & 2.08 \\
\hline 7 & $\mathrm{nC} 133$ & AATCAAACACACACCTTGCG & GCAAGGGAATAAGGTGACGA & 2 & 55 & 0.17 & 2.05 \\
\hline 8 & umc2104 & CTGCTGGCAGTGGCAGTATTC & TACTGCTACACCTTTGTCGTCACC & 2 & 55 & 0.40 & 3.00 \\
\hline 9 & bnlg1523 & GAGCACAGCTAGGCAAAAGG & CTCGCACGCTCTCTCTTCTT & 3 & 55 & 0.43 & 3.03 \\
\hline 10 & umc 1908 & CGTACACTCAATCACGATCCAAAC & AACTTTGGGTACAAGTCAAGA & 2 & 55 & 0.50 & 3.04 \\
\hline 11 & phi085 & AGCAGAACGGCAAGGGCTA & TTTGGCACACCACGACGA & 2 & 55 & 0.48 & 5.06 \\
\hline 12 & umc 1378 & GAAGTCGCTGATGAGAACGTAACC & GCTAGCTAGTGTGAGTTCTTCCGC & 2 & 55 & 0.11 & 7.00 \\
\hline 13 & phi112 & TGCCCTGCAGGTTCACATTGAGT & AGGAGTACGCTTGGATGCTCTTC & 3 & 55 & 0.11 & 7.01 \\
\hline 14 & umC1889 & CCAATGTGCTGACGAACCATT & TCACCCTCCTTCTATGTTGTCCAT & 2 & 55 & 0.26 & 8.05 \\
\hline 15 & umc1161 & GCTCGCTGTTGGTAGCAAGTTTTA & GGTACCGCTACTGCTTGTTACTGC & 2 & 55 & 0.37 & 8.06 \\
\hline 16 & bnlg128 & CACCTGGAGGGACCCATTCC & AGGACCACAGGATCCATCCATCATCCT & 3 & 55 & 0.49 & 9.07 \\
\hline 17 & umc1061 & AGCAGGAGTACCCATGAAAGTCC & TATCACAGCACGAAGCGATAGATG & 2 & 55 & 0.50 & 10.06 \\
\hline 18 & umc1249 & GACCAGCAGCACTAGAGGACATTT & CTTCTGTTACTTTGGCAGCGGTT & 2 & 55 & 0.56 & 10.10 \\
\hline & & & Average & 2.22 & & 0.36 & \\
\hline
\end{tabular}

Fig.1 Diversity among the maize genotypes amplified with primer set bnlg1523 along with a $50 \mathrm{bp}$ ladder (M). The lane sequence in the gel corresponds to the identification of the genotypes as indicated in Table 1

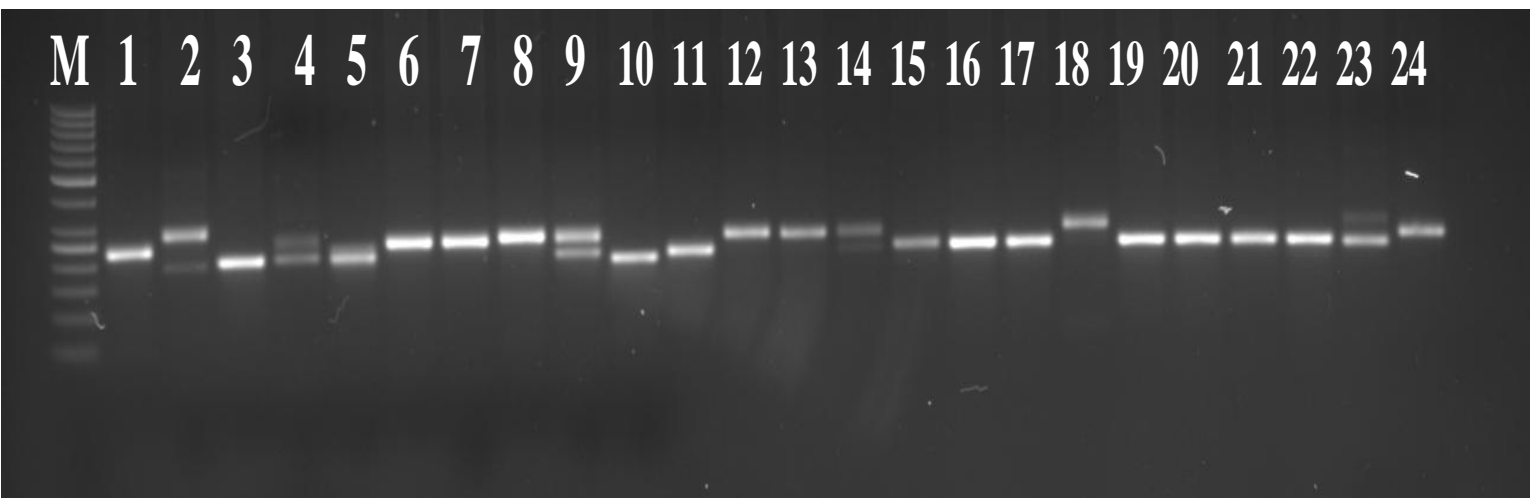

Fig.2 Diversity among the maize genotypes amplified with primer set phi033 along with a 50bp ladder (M). The lane sequence in the gel corresponds to the identification of the genotypes as indicated in Table 1

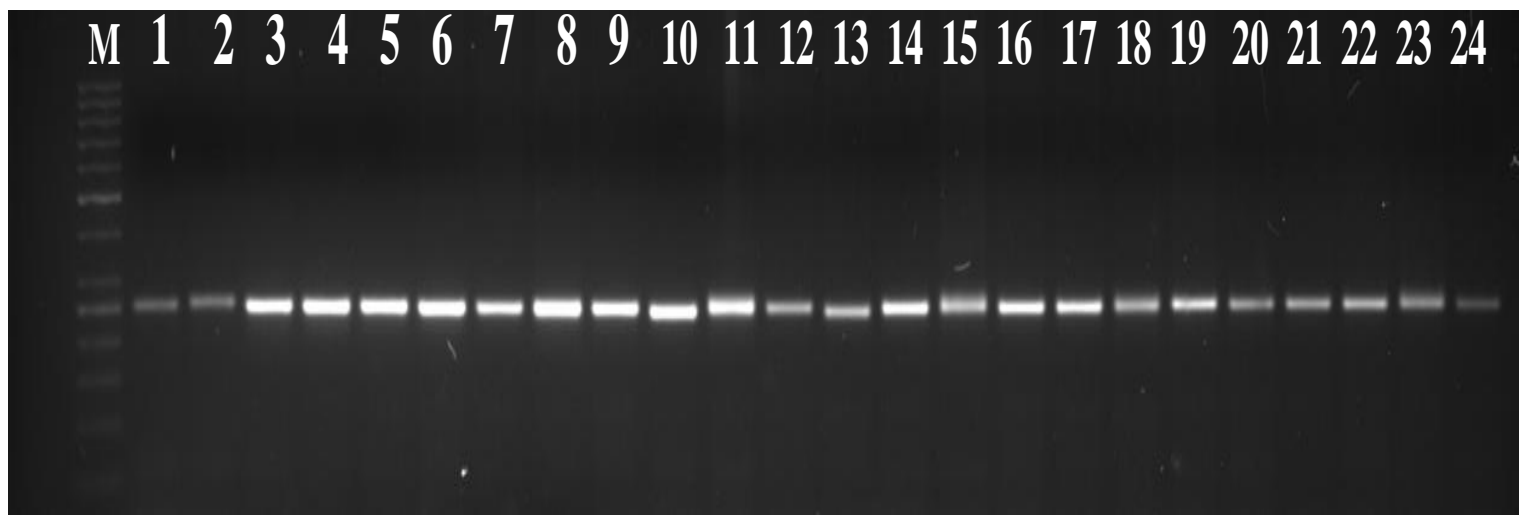


Fig.3 UPGMA cluster analysis grouped the genotypes into two major clusters. Cluster I was the biggest one with 25 genotypes which was further divided into two sub-clusters. The sequence in the cluster is corresponds to the identification of the genotypes as indicated in Table 1

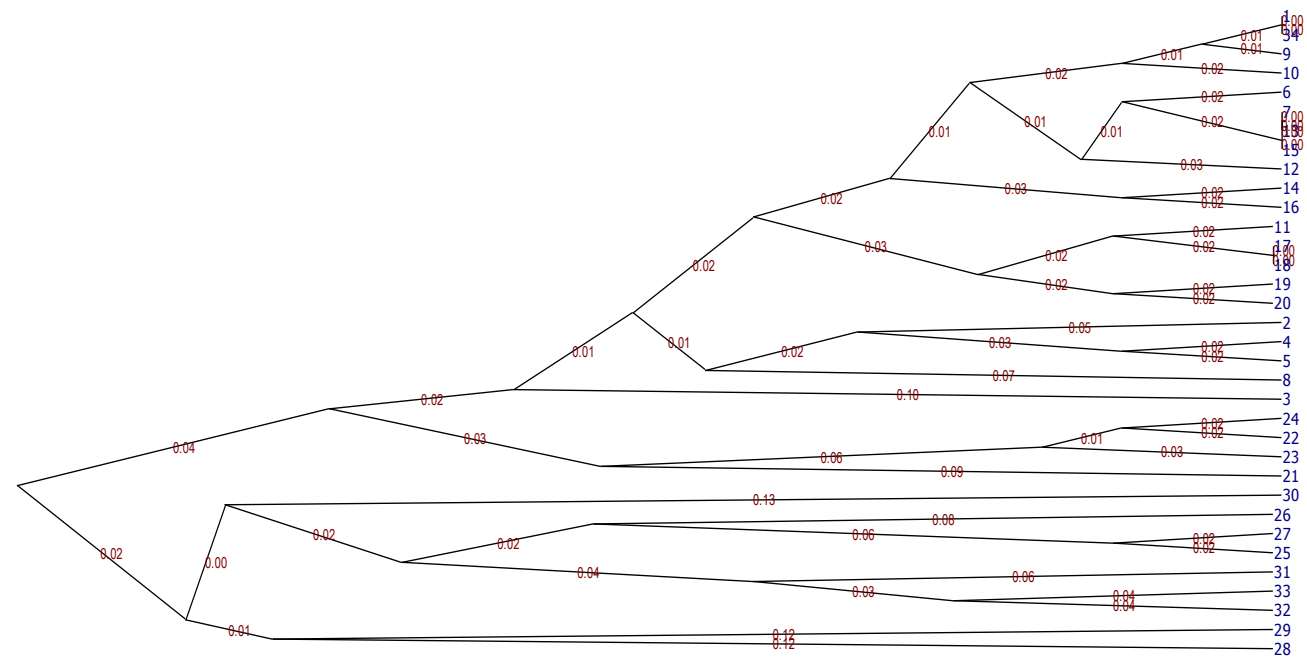

Realization of heterosis in any crop is dependent on the understanding of genetic diversity among the materials. The number of loci identified per microsatellite is a major limiting factor to detect genetic diversity among the lines studied. Other factors, like the repeat type and the methodologies employed for detection of polymorphic markers also influence allelic difference (Legesse et al., 2007). The average number of alleles (2.22), we observed in this study was considerably lower than those reported in maize by different researchers. Kumar et al., (2008) observed 2.96 in 16 popular inbred lines using 24 SSR markers. Warburton et al., (2002) found 4.9 alleles with 85 SSR markers. However, However, Bantte and Prasanna (2003) reported 3.25 alleles using 36 SSR loci. All these investigators used agarose gel system to screen the microsatellite loci, which reveals low allele number as compared to Poly acryl amide gel electrophoresis (PAGE) based screening. Agarose gel electrophoresis analysis being less cumbersome as compared to Poly acryl amide gel electrophoresis (PAGE) and less costly as compared to automated system, is most appropriate technology for routine analysis. However, resolving power of automated detection system and polyacrylamide gel is higher and thus number of alleles obtained would be higher than agarose based system. This becomes more relevant in case of SSR loci containing dinucleotide repeats. Laborda et al., (2005) detected 5.2 alleles per locus with 50 SSR markers using polyacrylamide gel. Amplicons differing in terms of two base pairs cannot be resolved using agarose gel (Legesse et al., 2007; Sibov et al., 2003). The lines used in the present investigation are less diverse in term of pedigree information as against other reports. Many of the lines have been derived from same base population. This may be the other reason for obtaining less average number of alleles (2.22) as compared to earlier reports. Genetic similarity as calculated by Jaccard's coefficient indicated considerable variability among the lines under study, therefore may be utilized in hybrids breeding programme. Further, the 18 polymorphic markers found out of total 25 ( $72 \%$ polymorphism), has also given an 
indication of unexploited variation in the selected lines. If the numbers of markers get increases, the variation can be exploited further. The genotypes belonging to two different clusters can be tried for development of high yielding maize hybrids. The study demonstrates the utility of SSR markers in determining genetic relationship among maize germplasm, which also have been demonstrated by various authors in recent past. Similar study taking more number of genotypes and more number of markers may give rise to better understanding of the situation.

\section{Acknowledgement}

First author acknowledged the laboratories facilities and guidance provided by ICARIIMR, Ludhiana.

\section{References}

Bantte, K. and Prasanna, B.M., 2003. Simple sequence repeat polymorphism in Quality Protein Maize (QPM) lines. Euphytica, 129(3):337-344.

Bar-Hen, A., Charcosset, A., Bourgoin, M. and Guiard, J., 1995. Relationship between genetic markers and morphological traits in a maize inbred lines collection. Euphytica., 84(2): 145154.

Berloo, R.V., 2008. GGT 2.0: versatile software for visualization and analysis of genetic data. J. Heredity, 99(2): 232236.

DAC, 2014. Agricultural Statistics at a Glance. Ministry of Agriculture, Government of India.

Jaccard, P., 1908. Nouvelles researches sur la distribution florale. Bull Soc. Vaud Sci. Na.t, 44:223-270.

Ko, W.R., Sa, K.J., Roy, N.S., Choi, H.J. and Lee, J.K., 2016. Analysis of the genetic diversity of super sweet corn inbred lines using SSR and SSAP markers. Genet. Mol. Res., 15(1).

Kumar, B., Rakshit, S., Singh, R.D., Gadag, R.N., Nath, R. and Paul, A.K., 2008. Genetic Diversity of Early Maturing Indian Maize (Zea mays L) Inbred Lines Revealed by SSR Markers. $J$. Plant Biochem. Biotechnol., 17(2):133140.

Laborda, P.R., Oliveira, K.M., Garcia, A.A.F., Paterniani, M.E.A.G.Z. and De Souza, A.P., 2005. Tropical maize germplasm: what can we say about its genetic diversity in the light of molecular markers? Theor Appl Genet, 111(7): 1288-1299.

Legesse, B.W., Myburg, A. A., Pixley, K. V., and Botha, A. M. 2007. Genetic diversity of African maize inbred lines revealed by SSR markers. Hereditas, 144(1): 10-17.

Lopes, A.D., Scapim, C.A., Machado, M.D.F.P.D., Mangolin, C.A., Silva, T.A., Cantagali, L.B., Teixeira, F.F. and Mora, F. 2015. Genetic diversity assessed by microsatellite markers in sweet corn cultivars. Scientia Agricola., 72(6):513-519.

Pushpavalli, S.N.C.V.L., Sudan, C., Singh, N.N. and Prasanna, B.M., 2001. Differentiation of elite Indian maize hybrids using simple sequence repeat markers. Indian J. Genet. $\mathrm{Pl} . \mathrm{Br}$., 61(4):304-308.

Reif, J.C., Hamrit, S., Heckenberger, M., Schipprack, W., Maurer, H.P., Bohn, M. and Melchinger, A.E., 2005. Trends in genetic diversity among European maize cultivars and their parental components during the past 50 years. Theor. Appl. Genet., 111(5):838-845.

Saghai-Maroof, M.A., Soliman, K.M., Jorgensen, R.A. and Allard, R.W., 1984. Ribosomal DNA spacer-length polymorphisms in barley: Mendelian inheritance, chromosomal location, and 
population dynamics. Proc Natl. Acad. Sci., 81(24): 8014-8018.

Senior, M.L. and Heun, M., 1993. Mapping maize microsatellites and polymerase chain reaction confirmation of the targeted repeats using a CT primer. Genome, 36(5): 884-889.

Sibov, S.T., Lopes De Souza Jr, C., GARCIA, A.A., GARCIA, A., Silva, A.R., Mangolin, C.A., Benchimol, L.L. and De Souza, A.P., 2003. Molecular mapping in tropical maize (Zea mays L.) using microsatellite markers. 1. Map construction and localization of loci showing distorted segregation. Hereditas, 139(2): 96-106.

Stacey J., Isaac P.G., 1994. Isolation of DNA from Plants. In: Isaac P.G. (eds) Protocols for Nucleic Acid Analysis by Nonradioactive Probes. Methods in
Molecular Biology. 28: 9-15. Humana Press. Totowa, NJ.

Stich, B., Melchinger, A.E., Frisch, M., Maurer, H.P., Heckenberger, M. and Reif, J.C., 2005. Linkage disequilibrium in European elite maize germplasm investigated with SSRs. Theor. Appl. Genet., 111(4): 723-730.

Warburton, M.L., Xianchun, X., Crossa, J., Franco, J., Melchinger, A.E., Frisch, M., Bohn, M. and Hoisington, D., 2002. Genetic characterization of CIMMYT inbred maize lines and open pollinated populations using large scale fingerprinting methods. Crop Sci. 42(6): 1832-1840.

XU, S.X., Liu, J.I.E. and LIU, G.S., 2004. The use of SSRs for predicting the hybrid yield and yield heterosis in 15 key inbred lines of Chinese maize. Hereditas, 141(3): 207-215.

\section{How to cite this article:}

Savita Sharma, D.P. Mishra, Bhupender Kumar, Harpreet Kaur and Jewlia, H.R. 2017. Genetic Diversity of Maize (Zea mays L.) Inbred Lines Revealed by Simple Sequence Repeat Markers. Int.J.Curr.Microbiol.App.Sci. 6(12): 543-550. doi: https://doi.org/10.20546/ijcmas.2017.612.066 